\title{
A hub covering network design problem for cargo applications in Turkey
}

\author{
S Alumur and BY Kara* \\ Bilkent University, Ankara, Turkey
}

\begin{abstract}
Hub location problems involve locating hub facilities and allocating demand nodes to hubs in order to provide service between origin-destination pairs. In this study, we focus on cargo applications of the hub location problem. Through observations from the Turkish cargo sector, we propose a new mathematical model for the hub location problem that relaxes the complete hub network assumption. Our model minimizes the cost of establishing hubs and hub links, while designing a network that services each origin-destination pair within a time bound. We formulate a single-allocation hub covering model that permits visiting at most three hubs on a route. The model is then applied to the realistic instances of the Turkish network and to the Civil Aeronautics Board data set.
\end{abstract}

Journal of the Operational Research Society (2009) 60, 1349-1359. doi:10.1057/jors.2008.92

Published online 8 October 2008

Keywords: hub location; hub cover; cargo applications; location; logistics

\section{Introduction}

Hubs are special facilities that serve as switching, transhipment, and sorting points in many-to-many distribution systems. Instead of serving each origin-destination pair directly, hub facilities consolidate flow in order to take advantage of economies of scale. Hub location problem involves locating hub facilities and allocating demand nodes to hubs in order to provide service between origin-destination pairs. There are various applications of the hub location problem in transportation, such as for air passengers and cargo and for telecommunication network design. In this study, we focus on cargo applications.

In a hub-network structure all demand points that generate flow are allocated to hub nodes. There are two basic types of allocation: single and multiple. In single allocation, non-hub nodes (demand points) are allocated only to a single hub; in multiple allocation, demand points can be allocated to more than one hub. Each demand point can send and receive flow only through the hub(s) that they are allocated to. In hub networks, it is assumed that economies of scale are incorporated by a discount factor (usually referred to as $\alpha$ ) when using hub-to-hub connections.

Hub location research began with the work of O'Kelly (1986). O'Kelly (1987) presented the first quadratic hub location model. His formulation is referred to as the single allocation $p$-hub median problem, because each demand centre is allocated to a single hub, $p$ hub facilities are to be

\footnotetext{
* Correspondence: BY Kara, Department of Industrial Engineering, Bilkent University, Bilkent, Ankara 06800, Turkey.

E-mail: bkara@bilkent.edu.tr
}

located, and the objective function minimizes the total transportation cost to serve a given set of demands. Later, various linearizations of this quadratic model were proposed in the literature (Campbell, 1994; Ernst and Krishnamoorthy, 1996; Skorin-Kapov et al, 1996). Among these models, the best mathematical formulation in terms of the computational time requirement is given by Ernst and Krishnamoorthy (1996), and the most efficient exact solution procedure is the shortest-path based branch-and-bound algorithm presented by Ernst and Krishnamoorthy (1998b). Researchers also considered the multiple allocation version of the problem, in which each demand centre may be allocated to more than one hub (Campbell, 1994; Skorin-Kapov et al, 1996; Ernst and Krishnamoorthy, 1998a).

In addition to considering the case when the number of hubs to be located is fixed, the hub location problem with fixed costs, in which the number of hubs to be located is a decision variable, also gained a considerable attention. Both single (O'Kelly, 1992; Ernst and Krishnamoorthy, 1999; Labbé et al, 2005; Cunha and Silva, 2007; Chen, 2007) and multiple allocation versions (Campbell, 1994; Mayer and Wagner, 2002; Marin et al, 2006; Cánovas et al, 2007) have been well studied. The reader may refer to Campbell et al (2002) and Alumur and Kara (2008) for recent reviews on hub location problems.

In the studies discussed above on the $p$-hub median problem and the hub location problem with fixed costs, the objective is to minimize the total transportation cost. The studies focusing on the service time are $p$-hub centre and hub covering type problems.

In the $p$-hub centre problem, the objective is to minimize the maximum travel time between two demand centres. This problem was first proposed by Campbell (1994). Kara 
and Tansel (2000) provided various linearizations, and Ernst et al (2002) provide the best mathematical formulation for the $p$-hub centre problem, up to now.

The hub covering problem is to locate hubs to cover all demand, such that the cost of opening facilities is minimized (Campbell, 1994). An origin-destination pair is thought to be covered if the travel time between them is within a predetermined time bound. Kara and Tansel (2003) studied this problem and proposed some new formulations. Different and better linear formulations are proposed by Ernst et al (2005) and Wagner (2008).

A few studies in the literature focus on hub location problems that arise in cargo applications. Kara and Tansel (2001) proposed a problem that they named the latest arrival hub location problem. Their objective was to minimize the arrival time of the last-arrived item, while accounting for both the flight times and the time spent at hubs for unloading, loading and sorting operations. Tan and Kara (2007) applied the latest arrival hub covering version to the cargo delivery sector in Turkey. Yaman et al (2007) incorporated multiple stopovers into the latest arrival hub centre model to determine vehicle routes.

In the hub location literature, it is usually assumed that the hub network is complete, with a direct link between every hub pair. However, building complete hub networks may unnecessarily increase the total investment costs in designing hub networks. In reality, most of the less-than-truckload and telecommunication networks do not operate on a complete hub network structure.

A few studies in the literature relax the complete hub network assumption. For example, the study by Nickel et al (2001) investigated the hub location problems arising in urban traffic networks and incurred a fixed cost for locating hub links. A recent study by Yoon and Current (2008) presented a model for the multiple allocation hub location and network design problem. They allowed direct connections between the non-hub nodes and also incurred variable arc costs associated with demand on the arcs. The hub arc location problems proposed by Campbell et al (2005a,b) presented a different modelling approach. Instead of locating hub facilities, their hub arc location problems located hub arcs with reduced unit costs. The resulting hub arc network in their problems need not be connected.

In this study, motivated from the cargo applications, a new hub location problem including hub network design decisions is proposed. The outline of this paper is as follows. The next section presents the motivation and definition of the problem. The third section presents and explains the proposed mathematical model. In the fourth section some linearizations of the model are introduced. The fifth section provides the computational analysis on both the Civil Aeronautics Board (CAB) data set-a benchmark data set from the literature - and the Turkish network, and the last section presents concluding remarks together with possible future research directions.

\section{Motivation and problem definition}

In an attempt to model the real-life hub location problems encountered in the cargo sector, in this paper, we aim to provide a tool for designing cost-effective hub networks for cargo companies. In order to observe the real-life requirements in this sector, many interviews were held with various cargo companies operating in Turkey. It was then found out that many of the hub location problems proposed in the literature lack some real-life requirements from this sector. In this section, we present our main observations from the cargo sector and then we will define our problem based on these observations.

In cargo applications, the transportation of cargo from origin to destination is handled by operation centres. The journey of a cargo starts from a branch office. A customer either takes his cargo to the branch office of a cargo firm or phones the firm for a pick-up. Branch offices are allocated to operation centres. At the end of each day, a branch office sends its whole cargo to its assigned operation centre. At the operation centres, the cargo is sorted according to the destination and is loaded into larger and more specialized vehicles based on the destination. When the cargo from every branch office allocated to that operation centre is received, the vehicles are sealed and start their routes. These routes are previously determined by the cargo company, so that at the end, each cargo is transported to the operation centre of its destination branch office. At the end of the journey, the branch offices pick up their cargo from the operation centres by themselves, and, finally, cargo reaches its destination point.

Because cargo companies use special, faster, and larger trucks travelling between operation centres, economies of scale are generated by this transportation. This structure used by cargo companies is precisely the same as the hub network structure. Therefore, we identify the branch offices of a cargo company as demand points and the operation centres as hubs. As each branch office is allocated to a single operation centre, in most of the cargo firms, we consider a single-allocation structure. In general this hub network structure is similar for most of the cargo companies; however, we note that each cargo company may have its own characteristics or requirements.

Through interviews with major cargo firms in Turkey, it was determined that the cargo firms' main objective is customer satisfaction. Customer satisfaction in this sector is directly related with reliability and guaranteed service time. In practice, the quicker and safer you send the cargo, the more likely the customers are to be satisfied. Most of the national and worldwide cargo companies operate on a time basis. They provide different services to customers based on different delivery time guarantees. Thus, in this sector, time is a major concern. Most of the hub location literature focuses on sending flows with minimum cost and does not consider service time at all. Conversely, in the models that consider time ( $p$-hub centre, hub covering, and latest arrival types of 
models), the transportation costs are neglected. However, in reality, both the establishment of hubs and using hub links incurs some cost, while guaranteeing service time. To the best of our knowledge no multi-objective study in the hub location literature considers the objectives related to both cost and service time. Because it was observed that service time is the primary objective for cargo companies, in this study, the service time is treated as a hard constraint rather than as an objective.

Truck synchronization is an important concern in designing hub networks for cargo companies. If a cargo truck is to visit a hub node in its route, then the flow generated from this inbetween hub may wait for that truck to arrive. Or conversely, the cargo may not be ready at a hub when the truck arrives. Given that there is an initial service time guarantee, a company needs to consider these waiting times so that the cargo is delivered within the promised service time.

In this study, while building our model, we focused on needs of a major cargo company operating in Turkey, say Company A. (The Company does not like to share either its name or the details of their hub network for confidentiality reasons.) Company A is among the largest cargo companies operating in Turkey. The company provides service between every city pair in Turkey. The company authorities believe that building hub facilities increases their service quality. Given that the company uses a high number of hubs, sending separate trucks from a hub to all other hubs is quite costly in terms of investment on the total number of trucks. Thus, they force some trucks to visit intermediate hubs to decrease this total investment cost. Hence, the company currently employs an incomplete hub network structure. Through our interviews with other companies in the region it was found that almost all of the cargo firms operating in Turkey do not employ a complete hub network structure. The incomplete hub network design problem is commonly encountered in the cargo sector. Therefore, the basic assumption in the hub location literature of building complete hub networks is not valid in these applications.

A general concern of the cargo companies is the safety of the cargo. Company A wants to ensure their customers that their cargo will arrive at the destination at the guaranteed time without any loss or damage to the cargo. For safety reasons, the cargo trucks travelling between hubs are sealed on the beginning of every route and unsealed at each stop at a hub. While using an incomplete hub network structure, a sealed truck can be unsealed at a hub other than the destination hub of a cargo in that truck. In these in-between stops at hubs a cargo may be mistakenly unloaded resulting in a delay in the service time or may get lost. Even though such instances are rarely met, many precautions are taken by the company to prevent any loss or delay of the cargo. One of their precautions is that they want to minimize the number of intermediate hub stops on any route. In a complete hub network structure, cargo trucks visit two hubs on a route. They are sealed in the origin hub and unsealed at the destination hub. In order to reduce the operational costs, while regarding safety, the Company A uses an incomplete hub network structure, where a cargo truck is allowed to make at most one additional stop in travelling between two hubs. So, they use a hub network on which each origin-destination pair receives service by visiting at most three hubs on a route.

With these observations, in this study, we propose a new mathematical model. This new model determines the location of hubs, allocates demand centres to these hubs, and designs a hub network by relaxing the assumption of having a fully interconnected hub network. We formulate a single-allocation hub covering model that permits visiting at most three hubs on a route. We have also considered the possible waiting times at the in between hub nodes while modelling the problem. The model minimizes the total costs, including the costs of establishing hubs and hub links, subject to a time limit on the maximum service time.

The proposed model is applicable for all the cargo companies operating on a time basis in addition to the ones operating in Turkey. By the use of our model it may be realized that designing complete hub networks is cost wise inefficient, while there is no contribution to the service time guarantee. On the other hand, using at most a 3-hub stop strategy rather than a complete (2-hub stop) one may decrease the investment on the total number of trucks considerably, while regarding safety. Since our model also takes the truck synchronization into account, it is possible to provide the same service, for example, to a network consisting of four hubs with four trucks in contrast to a complete hub network requiring 12 trucks.

A generalization of our problem is the incomplete hub covering network design problem. Incomplete hub networks are commonly encountered in many real-life hub network structures. Our 3-stop strategy is a special case of the incomplete hub network design problem. However, the 3-hub stop networks are desirable for many applications. For example, for the cargo companies using air transportation, employing complete hub networks may be cost wise inefficient since an airplane is to be assigned between every hub. On the other hand, taking off and landing constitute the main operational cost of a flight. So, in order to decrease operational costs it is desirable to limit the number of take offs and landings for each airplane, for example to three. In addition, there are some air regulations restricting the total flight time of an airplane and staff for a single route. Similarly, in air passenger applications, assigning separate aircraft and staff between every hub causes congestion in airports and air networks, as well as high investment and operating costs to the company. However, it is also very undesirable for the passengers to stop at four or five hubs on a route. So, airline companies need to limit the number of hub stops on a route as well.

Many additional special cases of building incomplete hub networks are proposed for different applications in the literature. For example, in telecommunication literature designing different hub network (usually referred as backbone network) topologies such as star, ring, tree, and path are considered. 
The reader may refer to Klincewicz (1998) for such applications. For some applications, it is desirable to use paths with few numbers of edges in telecommunication. Dahl (1999) and Dahl and Johannessen (2004) pointed out the need for using few edges in paths in order to avoid unacceptable delay and to increase reliability. The constraint on the number of edges to be visited in between any origin-destination pair is referred as hop-constraints. Dahl (1999) studied the $k$-hop constrained problem and the related polyhedra. Dahl and Johannessen (2004), on the other hand, studied the 2-hop constraint problem on a given network and proved its NP-hardness. They provide a path-based formulation of the problem and identified its polyhedral. The 2-hop constraint idea is very similar to our 3-hub stop idea. The former restricts the number of edges to be visited to two while we restrict the number of hub nodes to be visited to three.

For cargo applications, $k$-additional stop topology for the hub network can also be considered. For safety concerns and the geographical structure of Turkey, Company A uses a 1 -additional stop strategy. Our main motivation in this study is to design optimum hub networks with the 1-additional stop strategy and also to observe the effect of relaxing the complete hub network assumption on service requirements.

In this study, some computational analysis on the Turkish network is provided. The model was also tested on the $\mathrm{CAB}$ data set, which is a benchmark data set used for hub location problems. It was shown through application of the well-known $\mathrm{CAB}$ data set that, in some cases, there is no need for a complete hub network, even for the tightest values of service time requirements.

\section{Mathematical model}

We are given a network on which the set of nodes (demand centres) are identified. Our problem is to find the location of hub nodes, to allocate the demand nodes to the located hub nodes, and to determine which links are to be established between hub nodes in order to provide service within a given time bound and allowing for at most three hub stops on any route. Let $N$ be the set of demand nodes, and let $H$ be the candidate set of hub nodes. Candidate hub nodes can be among the set of demand nodes.

The parameters of our mathematical model are as follows:

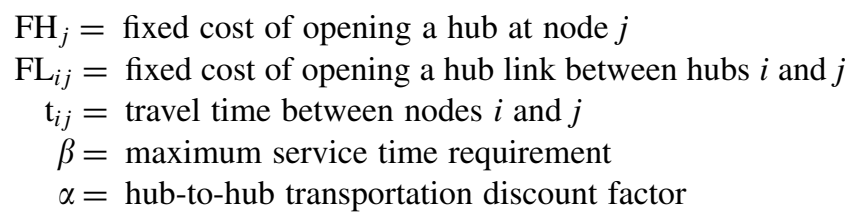

The decision variables of the mathematical model:

$$
\begin{aligned}
r_{j} & =\text { ready time of cargo at hub } j \\
x_{i j} & =1 \text { if node } i \text { is allocated to hub at node } j ; 0 \text { otherwise }
\end{aligned}
$$

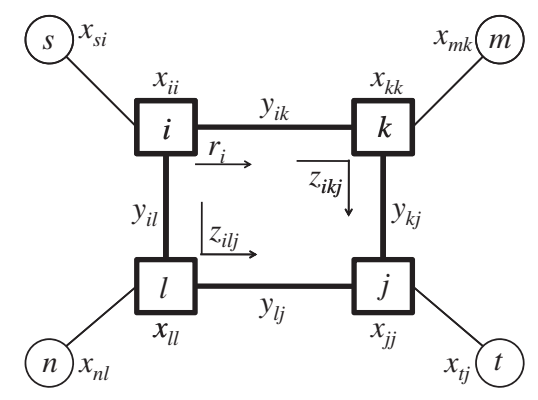

Figure 1 Decision variables of the mathematical model.

$y_{i j}=1$ if a hub link is established between hubs $i$ and $j$; 0 otherwise

$z_{i k j}=1$ if hub $k$ is used when travelling from hub $i$ to hub $j$; 0 otherwise

The decision variables of the model are schematically shown in Figure 1.

An integer programming formulation of the problem (3-stop-0) defined above is as follows:

$$
\text { Minimize } \sum_{j \in H} \mathrm{FH}_{j} x_{j j}+\sum_{j \in H} \sum_{i \in H} \mathrm{FL}_{i j} y_{i j}
$$

subject to

$$
\begin{array}{cc}
\sum_{j \in H} x_{i j}=1 & \text { for all } i \in N \\
x_{i j} \leqslant x_{j j} & \text { for all } i \in N, \quad j \in H \\
2 y_{i j} \leqslant x_{i i}+x_{j j} & \text { for all } i \in H, \quad j \in H \\
\left(1-y_{i j}\right)-\sum_{l \in H: l \neq i} x_{i l}-\sum_{l \in H: l \neq j} x_{j l} & \\
\leqslant \sum_{k \in H: k \neq i, k \neq j} z_{i k j} & \text { for all } i \in H, j \in H \\
2 z_{i k j} \leqslant y_{i k}+y_{k j} & \text { for all } i \in H, \\
\sum_{k \in H} z_{i k j} \leqslant\left(1-y_{i j}\right) & \text { for all } i \in H, \quad j \in H \\
& \text { for all } i \in H, \quad j \in H \\
y_{i j}=y_{j i} & \text { for all } i \in N, \quad j \in H \\
r_{j} \geqslant t_{i j} x_{i j} & \text { for all } i \in H, \quad j \in H \\
\left(r_{j}+r_{i}+\alpha t_{i j}\right) y_{i j} \leqslant \beta & \\
\left(r_{j}+\operatorname{Max}\left\{r_{k}, r_{i}+\alpha t_{i k}\right\}+\alpha t_{k j}\right) z_{i k j} \leqslant \beta \\
\text { for all } i \in H, \quad j \in H, \quad k \in H \\
x_{i j} \in\{0,1\} & \text { for all } i \in N, \quad j \in H \\
y_{i j} \in\{0,1\} & \text { for all } i \in H, \quad j \in H \\
z_{i k j} \in\{0,1\} & \text { for all } i \in H, \quad j \in H, \\
r_{j} \geqslant 0 & \text { for all } j \in H
\end{array}
$$

The objective function (1) minimizes the total cost of establishing the hub network. The total cost term includes the fixed 
cost of locating hubs and establishing hub links. Constraints (2) and (12) together ensure that each demand node is allocated to exactly one hub, that is, we use single-allocation. Constraint (3) states that a demand node can be allocated to a node only if a hub is opened at that node. Constraint (4) links $x$ variables to $y$ variables and ensures that a hub link can only be opened between two established hubs.

We force the model via constraint (5) so that if a direct link does not exist between two hub nodes, these two hubs must be reachable via stopping at a hub node in between. Thus, every two demand centre can receive service via at most three hubs on a route. Note that for given $i$ and $j$, the summations $\sum_{l \in H: l \neq i} x_{i l}$ and $\sum_{l \in H: l \neq j} x_{j l}$ on the left-hand side of the constraint (5) both take on the value 0 if $i$ and $j$ are both hub nodes, that is, if $x_{i i}=1$ and $x_{j j}=1$. Thus, the left-hand side of the constraint (5) takes on the value 1, if a direct hub link is not established between two established hubs and forces the $z$ variable to take on the value 1 for some hub $k$. Constraints (6) and (7) are logical constraints linking $y$ and $z$ variables. The in-between hub can only be used if a direct hub link exists from both of the hubs (constraint (6)). We do not need to use an in-between hub, if a direct hub link connection between two hubs exists, and exactly one hub must be used in travel between two hubs (constraint (7)).

The case for an in-between hub is illustrated in Figure 1. Because $y_{i j}=0$ for $x_{i i}=1$ and $x_{j j}=1$, the left-hand side of constraint (5) takes on the value 1 forcing the model to use another hub in between hubs $i$ and $j$. By constraints (6) and (7) $z_{i k j}$ must be equal to 1 for some $k$ such that $x_{k k}=1$, $y_{i k}=1$, and $y_{k j}=1$. Then in the figure either $z_{i k j}$ or $z_{i l j}$ must be equal to 1 . Note that by constraint (7) only one of $z_{i k j}$ or $z_{i l j}$ can take on the value 1 .

We establish an undirected hub network so that if a hub link is opened in one direction it should also be opened in the other direction (constraint (8)). Constraint (9) ensures that the ready time of the cargo at a hub is greater than the time needed to travel from all the demand points allocated to that hub. Remember that in cargo applications, a hub waits for all the cargo coming from demand centres that is allocated to that hub before sending the cargo to another hub or demand centre. The left-hand side of constraint (10) calculates the maximum travel time between demand centres allocated to two different hubs, when a direct hub link is established between these two hubs. Whereas, the maximum travel time between demand centres allocated to two different hubs when a direct hub link is not established in between is calculated in constraint (11). Remember that if there is not a direct hub link between two hubs, there is a known hub to be visited in between, which is obtained by $z$ variables. Note that the ready time of the cargo at the in-between hub may be greater than the time required to travel from the origin to the inbetween hub. Thus, we need the maximum operator on the left-hand side of constraint (11). Constraints (12)-(15) are the constraints that define binary variables and the non-negativity constraints.
This mathematical model is a nonlinear programming model due to constraints (10) and (11). If we let $|N|=n$ and $|H|=h$ the model has $\left(h^{3}+h^{2}+n h\right)$ binary variables and $\left(2 h^{3}+5 h^{2}+2 n h+n\right)$ constraints.

\section{Linearizations}

We propose constraint (10a) below for the linearization of constraint (10).

$$
r_{j}+r_{i}+\alpha t_{i j} y_{i j} \leqslant \beta \quad \text { for all } i \in H, \quad j \in H
$$

Let us refer to the new formulation by replacing constraint (10) with (10a) as (3-stop-1).

Theorem 1 Any feasible solution to (3-stop-0) is a feasible solution to (3-stop-1) and vice versa.

Proof Let $(\bar{x}, \bar{y}, \bar{z}, \bar{r})$ be a feasible solution to (3-stop-0). Let us show that $(\bar{x}, \bar{y}, \bar{z}, \bar{r})$ is also feasible to (3-stop-1). As all constraints other than constraint (10) are common to both, it suffices to show that $(\bar{x}, \bar{y}, \bar{z}, \bar{r})$ is feasible to (10a). Consider the Equation (10a) associated with nodes $i$ and $j$. There are two cases depending on the value of $y_{i j}$.

- Case 1: $y_{i j}=1$. Then constraints (10) and (10a) yield the same left-hand side.

- Case 2: $y_{i j}=0$. The left-hand side of the constraint (10) yields 0 ; however, the left-hand side of the constraint (10a) yields $r_{j}+r_{i}$. It suffices to show that $r_{j}+r_{i}$ is less than or equal to $\beta$. Note that when $i=j$, constraint (10a) yields $2 r_{i} \leqslant \beta$ because $t_{i i}=0$ and $y_{i i}=0$. Thus, we have both $r_{j} \leqslant \beta / 2$ and $r_{i} \leqslant \beta / 2$. By summing these two, we obtain $r_{j}+r_{i} \leqslant \beta$. Thus, constraint (10a) is satisfied.

To prove the converse, observe that the left-hand side of (10) is always less than or equal to the left-hand side of (10a); that is,

$\left(r_{j}+r_{i}+\alpha t_{i j}\right) y_{i j} \leqslant r_{j}+r_{i}+\alpha t_{i j} y_{i j} \quad$ for all $i \in H, \quad j \in H$.

Therefore, any feasible solution to (3-stop-1) is also feasible to (3-stop-0).

For the linearization of constraint (11), we provide two sets of constraints below:

$r_{j}+r_{k}+\alpha t_{k j} z_{i k j} \leqslant \beta \quad$ for all $i \in H, \quad j \in H, \quad k \in H$

$r_{j}+r_{i}+\alpha\left(t_{i k}+t_{k j}\right) z_{i k j} \leqslant \beta \quad$ for all $i \in H, \quad j \in H, \quad k \in H$

Let us refer to the new formulation by replacing constraint (11) with (11a) and (11b) in (3-stop-1) as (3-stop-2).

Theorem 2 Any feasible solution to (3-stop-1) is a feasible solution to (3-stop-2) and vice versa. 
Proof Let $(\bar{x}, \bar{y}, \bar{z}, \bar{r})$ be a feasible solution to (3-stop-1). Let us show that $(\bar{x}, \bar{y}, \bar{z}, \bar{r})$ is also feasible to (3-stop-2). Because all constraints other than constraint (11) are common to both models, it suffices to show that $(\bar{x}, \bar{y}, \bar{z}, \bar{r})$ is feasible to (11a) and (11b). Consider the Equation (11a) and (11b) associated with nodes $i, j$, and $k$. There are three cases, depending on the value of $r_{i}, r_{k}$, and $z_{i k j}$.

- Case 1: $z_{i k j}=1$

- Case 1a: $r_{k} \geqslant r_{i}+\alpha t_{i k}$. Then constraints (11) and (11a) yield the same left-hand side. However, the left-hand side of constraint (11b) yields $r_{j}+r_{i}+\alpha\left(t_{i k}+t_{k j}\right) \leqslant \beta$. But as $r_{j}+r_{i}+\alpha t_{i k}+\alpha t_{k j} \leqslant r_{j}+r_{k}+\alpha t_{k j} \leqslant \beta$, the constraint $(11 \mathrm{~b})$ is also satisfied.

- Case $1 b: r_{k}<r_{i}+\alpha t_{i k}$. Then constraints (11) and (11b) yield the same left-hand side. The left-hand side of constraint (11a) yields $r_{j}+r_{k}+\alpha t_{k j} \leqslant \beta$. But as, $r_{j}+$ $r_{k}+\alpha t_{k j}<r_{j}+r_{i}+\alpha t_{i k}+\alpha t_{k j} \leqslant \beta$, the constraint (11a) is also satisfied.

- Case 2: $z_{i k j}=0$. The left-hand side of the constraint (11) yields 0 ; however, the left-hand side of the constraint (11a) yields $r_{j}+r_{k}$, and the left-hand side of the constraint (11b) yields $r_{j}+r_{i}$. It suffices to show that both $r_{j}+r_{k}$ and $r_{j}+r_{i}$ are less than or equal to $\beta$. From constraint (10a) and the argument in the proof of Theorem 1 , we know that $r_{j} \leqslant \frac{\beta}{2}$, $r_{k} \leqslant \frac{\beta}{2}$ and $r_{i} \leqslant \frac{\beta}{2}$. By summing these constraints, we obtain $r_{j}+r_{k} \leqslant \beta$ and $r_{j}+r_{i} \leqslant \beta$. Thus, both constraints (11a) and (11b) are satisfied.

Thus, we conclude that $(\bar{x}, \bar{y}, \bar{z}, \bar{r})$ is also feasible to (3-stop-2).

To prove the converse, observe that the left-hand side of (11) is either equal to the left-hand side of (11a) or (11b) or less than both of them. So any feasible solution to (3-stop-2) is also feasible to (3-stop-1).

Now, let us state the linearized mathematical model (3-stop)

Minimize (1)

subject to (2)-(9), (10a), (11a), (11b), (12)-(15).
Corollary 1 Any feasible solution to (3-stop-0) is a feasible solution to (3-stop) and vice versa.

Corollary 2 An optimum solution to (3-stop) is also an optimum solution to (3-stop-0) and vice versa.

(3-stop) is a strong linearization of (3-stop-0) in three ways: (1) it uses precisely the same set of variables as in (3-stop-0), that is, there is no change in the dimension of the space; (2) the feasible sets are the exactly the same; and (3) the optimal sets are the same.

For our applications we added constraint (16):

$$
z_{i k j} \leqslant\left(1-y_{i j}\right) \quad \text { for all } i \in H, \quad j \in H, \quad k \in H
$$

to the model (3-stop) in order to have tighter LP relaxations.

\section{Computational results}

The model is first applied on the Turkish network. On this network, 81 cities are considered demand centres. We took 16 candidate sites for hub locations among these demand centres: the most populated and industrialized cities in Turkey suitable for hub location (Yaman et al, 2007). Figure 2 shows the geographical locations of the demand centres and candidate hub locations on a map of Turkey and Table 1 presents the names of the candidate hub locations.

Our problem parameters for this Turkish network are summarized in Table 2. The travel times $\left(t_{i j}\right)$ between all nodes on the network can be obtained from Kara (2008). The fixed costs for locating hub facilities $\left(\mathrm{FH}_{j}\right)$ are taken from a previous study by Tan and Kara (2007). Various factors, such as the industrialization level, the in and out cargo intensity, land price, and the highway intensity of different cities have been considered in determining these fixed costs.

In addition to the fixed cost of opening hubs, the total cost term in the objective function includes the costs for establishing hub links $\left(\mathrm{FL}_{i j}\right)$. In order to propose a general model, we allowed for the costs of establishing hub links to differentiate each link in the model. However, through our interviews with cargo firms we observed that the costs for

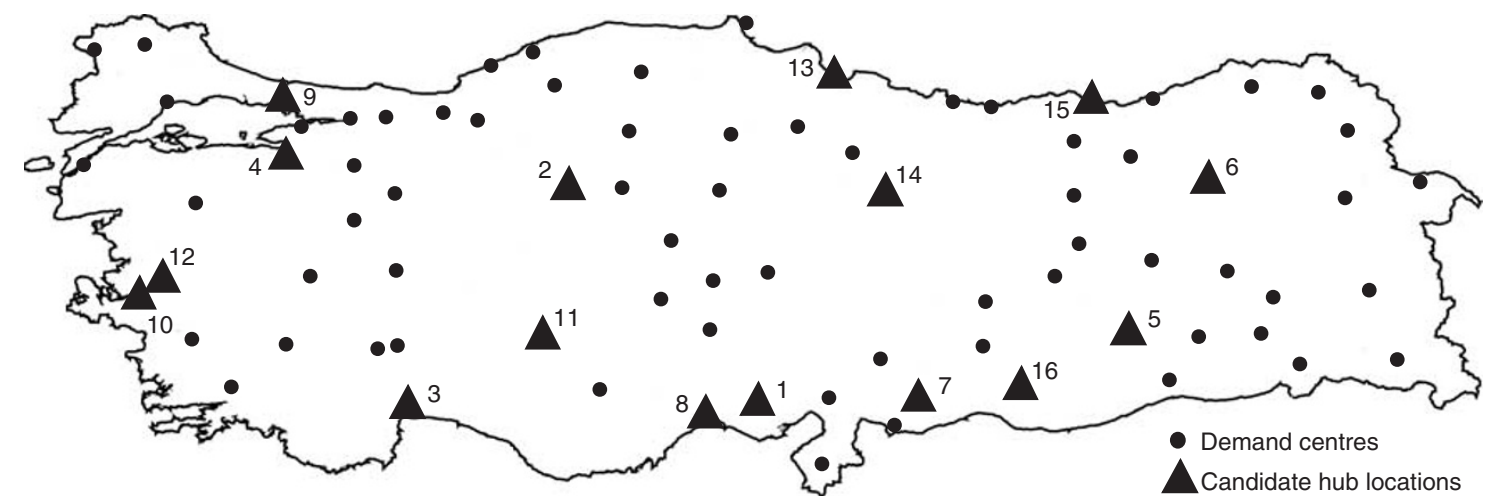

Figure 2 Geographical locations of the 81 demand centres, 16 of which are candidate hub locations. 
using inter-hub links are actually fixed, are the same for all links, and are not proportional to distances, that is, that $\mathrm{FL}_{i j}=$ FL for all $i \in H$ and $j \in H$. Thus, we take the link costs to be fixed in our computations. In order to observe the changes on the hub network with respect to these cost values, we took two different fixed cost values for hub links: low link cost and high link cost. The low link cost value is a fixed value that is taken as relatively lower than the average fixed hub costs, and the high link cost value is taken as approximately the average of fixed hub cost values.

Through our interviews with cargo firms, the hub-to-hub transportation time discount factor $(\alpha)$ was found to be 0.9 on ground transportation in Turkey. Thus, we took $\alpha$ to be 0.9 in all of our computations.

Table 1 Names of the candidate hub locations

\begin{tabular}{llll}
\hline 1. Adana & 5. Diyarbakır & 9. İstanbul & 13. Samsun \\
2. Ankara & 6. Erzurum & 10. İzmir & 14. Sivas \\
3. Antalya & 7. Gaziantep & 11. Konya & 15. Trabzon \\
4. Bursa & 8. Mersin & 12. Manisa & 16. Şanliurfa \\
\hline
\end{tabular}

Table 2 Parameters for the Turkish network

\begin{tabular}{lc}
\hline Parameter & Value \\
\hline$|N|$ & 81 \\
$|H|$ & 16 \\
$t_{i j}, \mathrm{FH}_{j}$ & From Tan and Kara (2007) \\
$\alpha$ & 0.9 \\
$\mathrm{FL}$ & Low and High \\
$\beta$ (min) & \\
Interval-1: 5 hubs & $1800-1820$ \\
Interval-2: 4 hubs & $1830-1850$ \\
Interval-3: 3 hubs & $1860-1920$ \\
Interval-4: 2 hubs & $1930-1980$ \\
\hline
\end{tabular}

In this Turkish network, with a 0.9 discount factor, the tightest possible service time value between two demand centres is about $30 \mathrm{~h}$, that is, $1800 \mathrm{~min}$. We varied the service time values $(\beta)$ between 30 and $33 \mathrm{~h}(1800-1980 \mathrm{~min})$ with 10 -min time intervals. In order to comment on the computational times more realistically, we divided the $\beta$ values into four intervals. The first interval (Interval-1) starts from the $\beta$ value of $1800 \mathrm{~min}$, which results in opening five hubs on the Turkish network. All of the tested $\beta$ values in Interval-1 lead to opening five hubs. Interval-2 starts from the first $\beta$ value leading to opening four hubs, which is $1830 \mathrm{~min}$. Thus, with 10 -minute time intervals, $1830 \mathrm{~min}$ is the tightest possible $\beta$ value for opening four hubs on this network. Similarly, the $\beta$ values in Interval-3 and Interval-4 lead to opening three and two hubs, respectively. The summary of the $\beta$ values and intervals are listed in Table 2.

We took our runs on CPLEX 8.1, on an AMD Opteron 252, $2.6 \mathrm{GHz}$ server with 2GB RAM. All the runs are solved to optimality.

Figures 3a-d schematically show some of our computational results. The location of the hubs and established hub links are shown in these figures. In order to avoid complications, we did not show the allocation of demand nodes in these figures. From Figure $3 a$ and $b$, with the service time bound of $1800 \mathrm{~min}$, observe that even though the link costs are different, there is no change in either the location of the hubs or the established hub links. However, note that the resulting hub network in both of the solutions is incomplete. In Figure 3c, we obtained a complete hub network with $1850 \mathrm{~min}$ of service time bound and with low link costs. On the other hand, when we increased the link costs (Figure 3d) the hubs are opened in different locations in order to reduce the total link cost, and we obtained an incomplete hub network. In general we discovered that, except in a few instances, our solutions on the Turkish network were insensitive to the link costs.
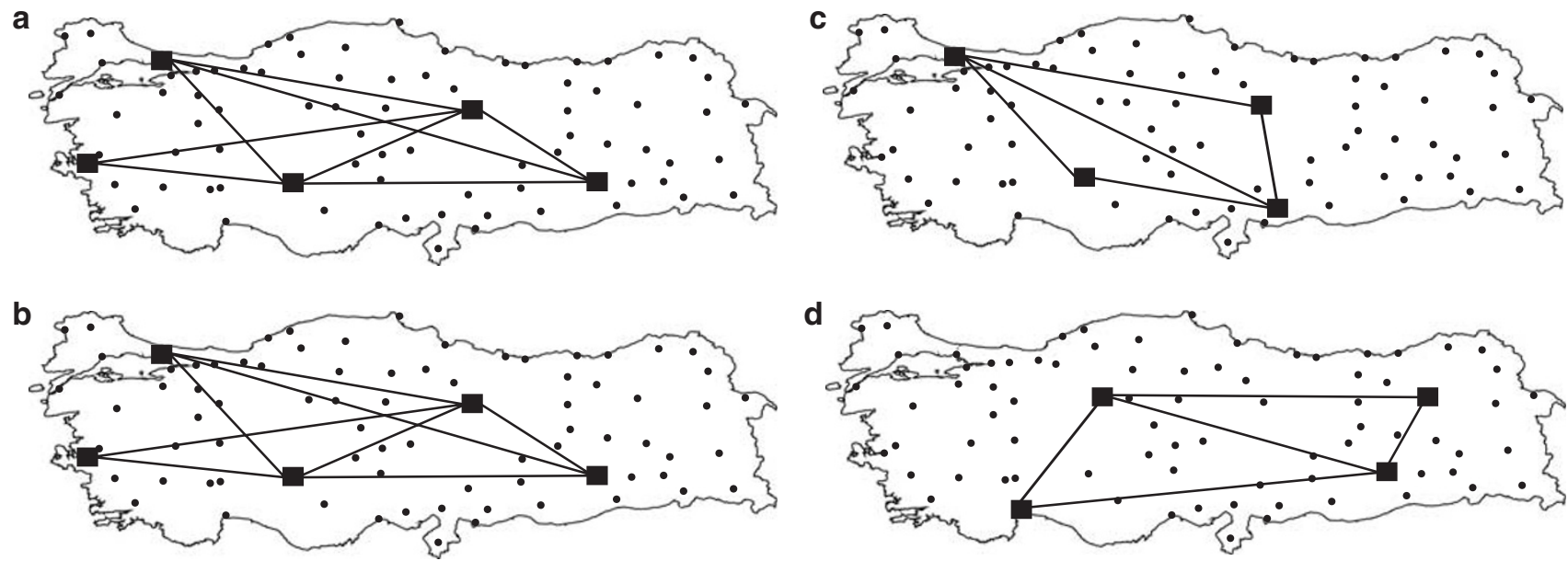

Figure 3 Computational results on the Turkish network. (a) $1800 \mathrm{~min}$, low link cost; (b) $1800 \mathrm{~min}$, high link cost; (c) $1850 \mathrm{~min}$, low link cost; and (d) 1850 min, high link cost. 
Table 3 shows our computational times obtained by using CPLEX 8.1. This table lists the minimum, maximum, and average CPU times obtained, corresponding to four different $\beta$ intervals. In Interval-1, we have an instance that lasted more than $7.5 \mathrm{~h}$. This is the highest CPU time that we have observed on this network, and it corresponds to the instance with the tightest $\beta$, shown in Figure $3 \mathrm{~b}$. However, even for Interval-1 with tight $\beta$ values we have results in an average of about $2.5 \mathrm{~h}$. This value decreases to approximately $45 \mathrm{~s}$ with loose $\beta$ values in Interval-4.

We have shared and discussed these solutions with different possible fixed costs and $\beta$ values with the authorities in Company A. Our proposed solutions turned out to be very different than their current hub network structure. The authorities in Company A are currently evaluating our solutions.

In order to discuss and compare our results with the hub location literature, we have also tested our model on the wellknown $\mathrm{CAB}$ data set. The CAB data set was first introduced by O'Kelly (1987), and it is based on the airline passenger interactions between 25 US cities in 1970, evaluated by the $\mathrm{CAB}$. Figure 4 shows the names and geographical locations of these 25 cities on the CAB data set.

The Parameters taken for the instances on the $\mathrm{CAB}$ data set are listed in Table 4 . There are 25 nodes in the CAB network,

Table 3 CPU times on the Turkish network

\begin{tabular}{lclc}
\hline & Min & \multicolumn{1}{c}{ Max } & Average \\
\hline$\beta$ Interval-1 & $43.13 \mathrm{~min}$ & $7.79 \mathrm{~h}$ & $2.47 \mathrm{~h}$ \\
$\beta$ Interval-2 & $19.38 \mathrm{~min}$ & $1.22 \mathrm{~h}$ & $43.46 \mathrm{~min}$ \\
$\beta$ Interval-3 & $1.34 \mathrm{~min}$ & $6.23 \mathrm{~min}$ & $2.96 \mathrm{~min}$ \\
$\beta$ Interval-4 & $7.62 \mathrm{~s}$ & $1.27 \mathrm{~min}$ & $45.47 \mathrm{~s}$ \\
\hline
\end{tabular}

and we took all nodes to be the candidate hub locations. Because there are no real travel-time values presented in the literature on the $\mathrm{CAB}$ data set, we took travel times equal to travel distances. Travel distances between 25 nodes on the CAB data set were obtained from Beasley (1990). Again, no real data on fixed hub costs is reported in the literature, so we took the fixed hub cost value to be 100 and fixed for all locations (O'Kelly, 1992). To observe any changes on the hub network we tested two different fixed link cost values. We took fixed link costs to be 1 and 100, where 1 corresponds to low link costs and 100 to high.

Similar to all of the applications on the CAB data set in the literature, we varied the $\alpha$ values. We took $\alpha$ to be $0.8,0.6,0.4$, and 0.2. We varied $\beta$ according to the optimum $p$-hub centre solutions found in Kara and Tansel (2001), corresponding to locating four, three, and two hubs for each $\alpha$ value. We again took our runs on CPLEX 8.1 on the same server.

Figure 5 presents two results from the $\mathrm{CAB}$ data set. In both of these results, the $\alpha$ value is taken to be 0.8 , and the $\beta$ value is the tightest possible service distance on this

Table 4 Parameters for the CAB network

\begin{tabular}{lc}
\hline Parameter & Value \\
\hline$|N|$ & 25 \\
$|H|$ & 25 \\
$t_{i j}$ & $=\mathrm{d}_{i j}$ \\
FH & 100 \\
FL & 1,100 \\
$\alpha$ & $0.8,0.6,0.4,0.2$ \\
$\beta$ & Tightest possible distance for each $\alpha$ value \\
& corresponding to locating four, three and two hubs \\
\hline
\end{tabular}

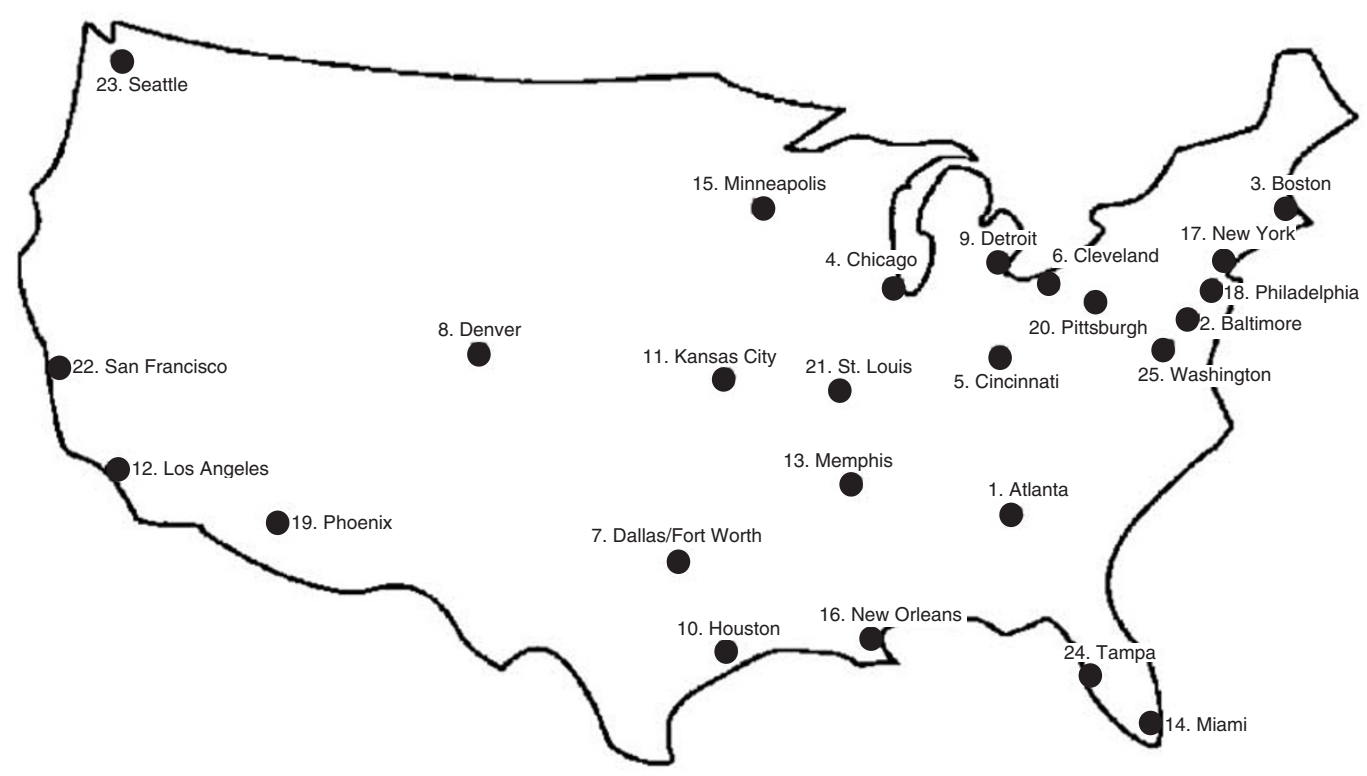

Figure 4 Names and geographical locations of the cities in the CAB data set. 

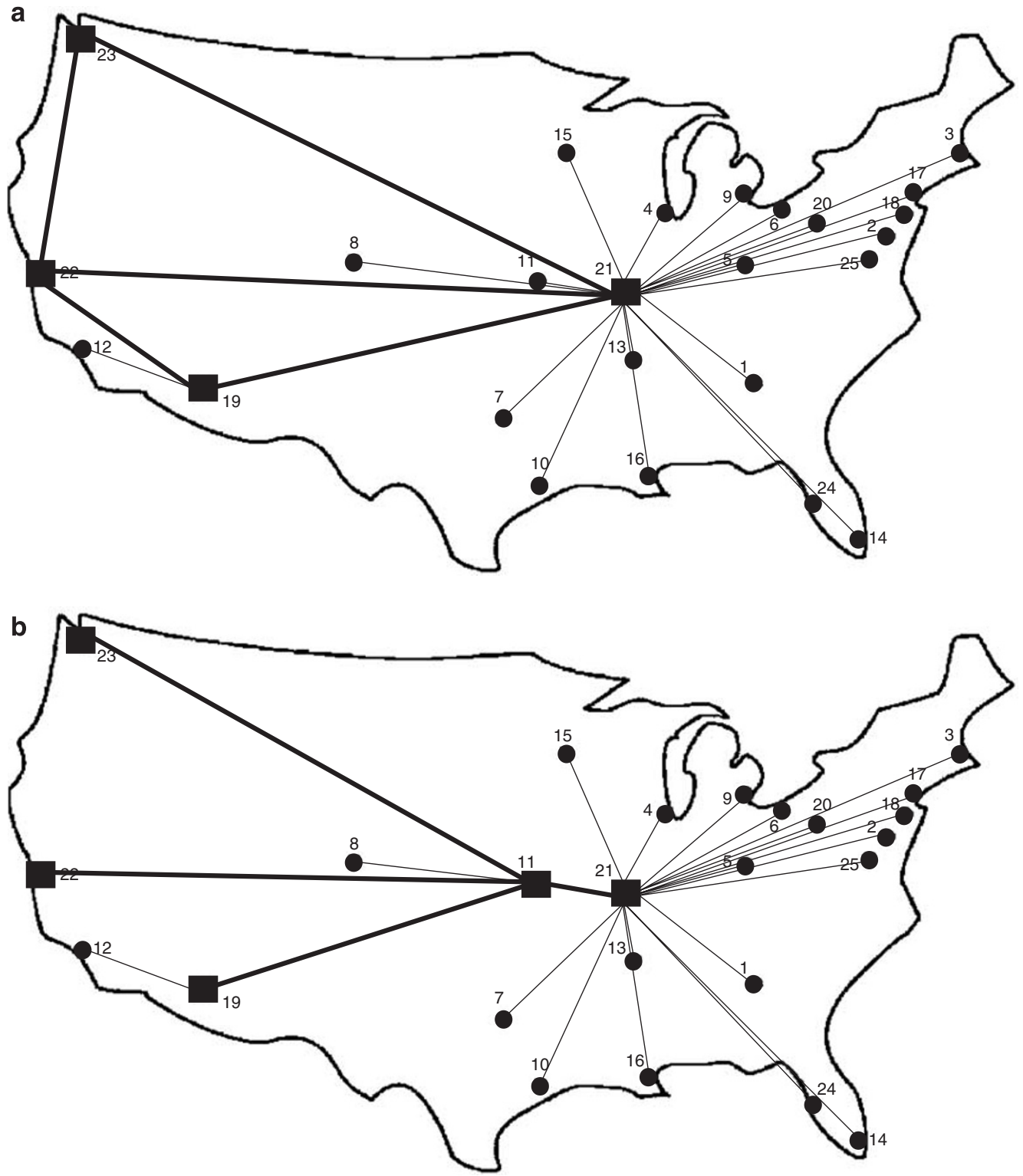

Figure 5 Computational results on the $\mathrm{CAB}$ data set. (a) $\alpha=0.8, \beta=2457$ with low link cost and (b) $\alpha=0.8, \beta=2457$ with high link cost.

network with four hubs, corresponding to this $\alpha$ value, which is 2457. Figures $5 \mathrm{a}$ and $\mathrm{b}$ show the corresponding results with low and high link costs, respectively. When we increased the fixed link costs, the model locates one more hub, and the link number is reduced by one. Note that in both of these figures the resulting hub network is incomplete, even though the service distance requirement is at its minimum possible value. The results from the $\mathrm{CAB}$ data set also prove that the complete hub network assumption presented in most of the hub location models is not necessary in application. In all of the $\mathrm{CAB}$ data set instances that corresponded to opening four hubs, all solutions resulted in incomplete hub networks.

We have listed our computational times on the $\mathrm{CAB}$ data set in Table 5. For each $\alpha$ value $(0.8,0.6,0.4$, and 0.2$)$ we
Table 5 The average CPU times for the CAB data set

\begin{tabular}{lcc}
\hline & Low link cost & High link cost \\
\hline$\alpha=0.2$ & $4.08 \mathrm{~min}$ & $1.05 \mathrm{~h}$ \\
$\alpha=0.4$ & $5.69 \mathrm{~min}$ & $20.93 \mathrm{~min}$ \\
$\alpha=0.6$ & $17.03 \mathrm{~min}$ & $50.36 \mathrm{~min}$ \\
$\alpha=0.8$ & $52.14 \mathrm{~min}$ & $6.32 \mathrm{~h}$ \\
\hline
\end{tabular}

took three different $\beta$ values and two different link costs (low and high).

In the $\mathrm{CAB}$ data set, the problems with low link costs tend to be solved quicker than the corresponding high linkcost instances. Also, the solution times tend to increase as $\alpha$ increases. The worst case performance of our model on 
this data set is obtained when the value of $\alpha$ is 0.8 . The highest CPU time that we observed on this data set is below $11 \mathrm{~h}$ (for the instance shown in Figure 5b), and the lowest is about $26 \mathrm{~s}$.

With both the Turkish network and the CAB data set, we have obtained optimal solutions with our proposed model in reasonable CPU times. Since we have tested the tightest possible $\beta$ values in both of the data sets, we presume that these are among the hardest instances on these data sets. When we compare the average $\mathrm{CPU}$ time requirements, the $\mathrm{CAB}$ data set instances turned out to be a little bit harder than the Turkish network instances. This is due to the increase in the number of candidate hub locations; the CAB data set contains 25 candidate hub locations, whereas the Turkish network contains 16 . On the other hand, we observed that the increase in the number of demand centres did not lead to a significant increase in the CPU time requirements compared to the increase in the CPU time with the increase in the number of candidate hub locations. In both of the data sets, except in a few instances, the solutions turned out to be insensitive to the link costs. However, again in both of the data sets, the low link cost instances required less CPU time than the corresponding high link cost instances.

Even though we have tested the tightest possible $\beta$ values in both of the data sets, the model resulted in building incomplete hub networks in most of the instances. This shows that the service that is provided with a complete hub network can also provided with an efficiently designed incomplete one with less investment cost requirements.

\section{Conclusion and future research directions}

After interviewing many cargo companies, many real-life requirements were observed from the cargo sector. It was found that the basic assumption in the hub location literature of building complete hub networks is not always valid. In this paper, this assumption is relaxed and a new mathematical formulation is proposed for a special case of the hub location problem for cargo applications. Because it was observed that time is a major concern the focus was on the hub covering version of the problem and a service time bound is considered for every origin-destination pair. In addition to the fixed costs of building hubs, the fixed costs of building hub links are also considered in the model. In order to take the waiting time at hubs into account, the truck synchronization is modelled as well.

The model designs an incomplete hub network allowing at most three hub stops on a route from any origin to destination. It has been observed that such a strategy is also desirable in air cargo, air passenger, and telecommunication networks, in addition to ground cargo transportation. After determining the link costs and service time requirements, a company can adopt the model presented in this study.

The model is solved in reasonable CPU times with the optimization solver CPLEX on both the Turkish network and the $\mathrm{CAB}$ data set. Some of the results on both networks are demonstrated. It was found that there is no need for a complete hub network even for the tightest values of service time requirements.

A generalization of the model is to build incomplete hub networks with no restriction on the number of hub stops. Reader should note that such networks may result in undesirably long paths in the hub network, causing probably higher operational costs. In addition to building general incomplete hub networks, one may focus on different special network structures applicable for different application areas of the problem such as for telecommunication applications.

It has been observed that real life has many additional requirements to those considered in this paper. For future research, one could focus on different objective functions. For example, one could maximize the next-day delivery between two demand centres. Furthermore, the multi-modal transportation problem is an important area of research. Today, most of the cargo companies employ both air and ground transportation networks, and we believe that the literature does not contain enough studies of this problem. Thus, there will be a need for hub location models in this area in the near future.

\section{References}

Alumur S and Kara BY (2008). Network hub location problems: The state of the art. Eur J Opl Res 190: 1-21.

Beasley JE (1990). OR-Library: Hub location. http://people.brunel.ac. uk/ mastjjb/jeb/orlib/phubinfo.html. Accessed 2 June 2008. Site last updated February 2008.

Campbell JF (1994). Integer programming formulations of discrete hub location problems. Eur J Opl Res 72: 387-405.

Campbell JF, Ernst AT and Krishnamoorthy M (2002). Hub location problems. In: Drezner Z and Hamacher HW (eds). Facility Location: Applications and Theory. Springer: Berlin, pp 373-407.

Campbell JF, Ernst AT and Krishnamoorthy M (2005a). Hub arc location problems: Part I-Introduction and Results. Mngt Sci 51: $1540-1555$.

Campbell JF, Ernst AT and Krishnamoorthy M (2005b). Hub are location problems: Part II-Formulations and Optimal Algorithms. Mngt Sci 51: 1556-1571.

Cánovas L, García S and Marín A (2007). Solving the uncapacitated multiple allocation hub location problem by means of a dual-ascent technique. Eur J Opl Res 179: 990-1007.

Chen JF (2007). A hybrid heuristic for the uncapacitated single allocation hub location problem. Omega 35: 211-220.

Cunha CB and Silva MR (2007). A genetic algorithm for the problem of configuring a hub-and-spoke network for a LTL trucking company in Brazil. Eur J Opl Res 179: 747-758.

Dahl G (1999). Notes on polyhedra associated with hop-constrained paths. Opns Res Lett 25: 97-100.

Dahl G and Johannessen B (2004). The 2-path network problem. Networks 43: 190-199.

Ernst AT and Krishnamoorthy M (1996). Efficient algorithms for the uncapacitated single allocation $p$-hub median problem. Location Sci 4: 139-154.

Ernst AT and Krishnamoorthy M (1998a). Exact and heuristic algorithms for the uncapacitated multiple allocation $p$-hub median problem. Eur J Opl Res 104: 100-112. 
Ernst AT and Krishnamoorthy M (1998b). An exact solution approach based on shortest-paths for $p$-hub median problems. Informs $J$ Comput 10: 149-162.

Ernst AT and Krishnamoorthy M (1999). Solution algorithms for the capacitated single allocation hub location problem. Ann Opl Res 86: $141-159$.

Ernst A, Hamacher H, Jiang H, Krishnamoorthy $M$ and Woeginger $\mathrm{G}$ (2002). Uncapacitated single and multiple allocation $p$-hub center problems. Unpublished report, CSIRO Mathematical and Information Sciences, Australia.

Ernst AT, Jiang H, Krishnamoorthy M (2005). Reformulations and computational results for uncapacitated single and multiple allocation hub covering problems. Unpublished report, CSIRO Mathematical and Information Sciences, Australia.

Kara BY (2008). Personal web site: Hub location. http://www.bilkent. edu.tr/ bkara/hubloc.htm. Accessed 2 June 2008. Site last updated December 2007.

Kara BY and Tansel BC (2000). On the single-assignment $p$-hub center problem. Eur J Opl Res 125: 648-655.

Kara BY and Tansel BC (2001). The latest arrival hub location problem. Mngt Sci 47: 1408-1420.

Kara BY and Tansel BC (2003). The single-assignment hub covering problem: Models and linearizations. J Opl Res Soc 54: 59-64.

Klincewicz JG (1998). Hub location in backbone/tributary network design: A review. Locat Sci 6: 307-335.

Labbé M, Yaman H and Gourdin E (2005). A branch and cut algorithm for hub location problems with single assignment. Math Program 102: 371-405.

Marin A, Cánovas L and Landete M (2006). New formulations for the uncapacitated multiple allocation hub location problem. Eur $J$ Opl Res 172: 274-292.
Mayer G and Wagner B (2002). HubLocater: An exact solution method for the multiple allocation hub location problem. Comput OR 29 : $715-739$.

Nickel S, Schobel A and Sonneborn T (2001). Chapter 1: Hub Location problems in urban traffic networks. In: Niittymaki J and Pursula M (eds). Mathematics Methods and Optimization in Transportation Systems. Kluwer Academic Publishers: Dordrecht.

O'Kelly ME (1986). The location of interacting hub facilities. Transport Sci 20: 92-105.

O'Kelly ME (1987). A quadratic integer program for the location of interacting hub facilities. Eur J Opl Res 32: 393-404.

O'Kelly ME (1992). Hub facility location with fixed costs. Pap Reg Sci 71: 293-306.

Skorin-Kapov D, Skorin-Kapov J and O'Kelly M (1996). Tight linear programming relaxations of uncapacitated $p$-hub median problems. Eur J Opl Res 94: 582-593.

Tan PZ and Kara BY (2007). A hub covering model for cargo delivery systems. Networks 49: 28-39.

Wagner B (2008). Model formulations for hub covering problems. $J$ Opl Res Soc 59: 932-938.

Yaman H, Kara BY and Tansel BC (2007). The latest arrival hub location problem for cargo delivery systems with stopovers. Transport Res Part B 41: 906-919.

Yoon M-G and Current J (2008). The hub location and network design problem with fixed and variable arc costs: Formulation and dualbased solution heuristic. J Opl Res Soc 59: 80-89.

Received November 2007; accepted June 2008 after two revisions 